\title{
The trials and tribulations of CAR-T cell development for cancer and AIDS
}

\author{
Kristen Hege is corporate vice president of translational medicine at Celgene and a clinical professor at University \\ of California, San Francisco (UCSF). She serves on the board of the Society for Immunotherapy of Cancer and was \\ recognized by FierceBiotech as one of the top women in biopharma.
}

\section{Kristen Hege}

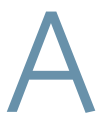
s I was sitting in a biosafety level 3 (BSL-3) lab at UCSF conducting an assay on HIV-infected blood, it hit me-this was not the path for me. At the time I was a junior faculty member working in an HIV lab, applying for grants and caring for patients with blood cancers. Meanwhile, my brother was next door at San Francisco General Hospital, dying of an HIV-related lymphoma, as were so many other young gay men with AIDS in San Francisco in the 1990s. I was his caregiver as both of my parents were dead; my mother died after a long battle with multiple sclerosis when I was in high school, and my father after an explosive battle with gastric cancer during my first semester of medical school. I was also a new parent of a 6-month-old daughter, fragile but full of promise.

A week before, I had spoken with Steve Sherwin, CEO of Cell Genesys, where I had spent two years during my hematology/ oncology fellowship researching how to engineer the immune system to kill cancer and HIV. Sherwin was preparing to launch the first ever clinical trial of chimeric antigen receptor (CAR)-T cells targeting HIV in collaboration with Clifford Lane at the US National Institutes of Health and needed a doctor to lead this and other cancer-directed CAR-T cell programs to follow. This wasn't the traditional academic medical career I had envisioned, but the translational focus felt right. I couldn't imagine giving up patient care, however, and I knew that Steve, still a part-time attending oncologist at UCSF, could relate. A clarity of purpose began to crystalize: too many diseases were fatal, and new treatments for cancer and AIDS were desperately needed. I accepted Sherwin's offer and negotiated an ' $80 / 20$ ' split, whereby $80 \%$ of my time would be spent on translational research at Cell Genesys and $20 \%$ on patient care at UCSF; in 1996, I embarked on my new hybrid career.

The early days of CAR-T cell clinical research were full of excitement and

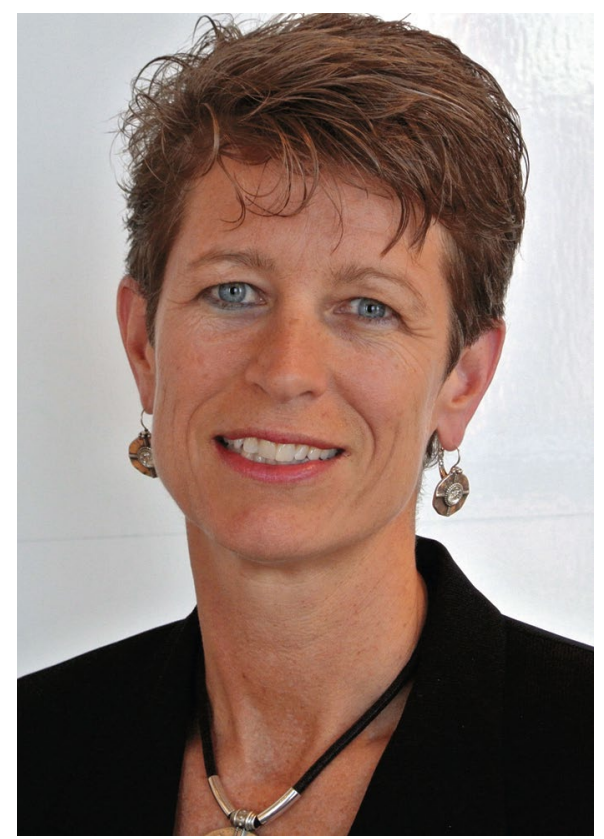

Credit: Kristen Hege

setbacks. As more effective HIV drugs were developed, hopes for a cure were high, and interest in immunotherapy waned. The focus shifted to cancer. We launched the first CAR-T cell trial for solid tumors (using CC49ל CAR-T cells) in 1996. While some degree of cell expansion and trafficking was demonstrated, the construct was immunogenic, and no tumor responses were observed. A patient's death in a gene therapy trial in 1999 set the entire field back, and biotech funding evaporated. Still, we learned some valuable lessons, including how to efficiently transduce T cells using kat (an acronym for 'kick ass transduction') retroviral vectors, how to optimize CAR-T cell function using ex vivo culture with anti-CD3 and anti-CD28 immunomagnetic beads (in collaboration with Carl June) and a combination of CD4 and CD8 T cells and, importantly, the safety of this gene therapy following decade-long CAR-T cell persistence in treated patients.

In 2010, I moved to Celgene to build a translational medicine program. Timing is everything; in 2012 the story broke that 11-year-old Emily Whitehead had achieved a complete remission of refractory leukemia after receiving CD19-targeted CAR-T cells. I reconnected with Mitch Finer from Cell Genesys, now CSO at bluebird bio. At the time, autologous, gene-modified cell therapy was far afield from Celgene's core strength in off-the-shelf oral cancer drugs. bluebird bio, meanwhile, had deep expertise in gene therapy, but no experience with cancer drug development. In 2013, our companies announced a partnership to develop CAR-T cells for cancer using bluebird's lentiviral vector platform.

Over the next several years, we selected our target, B cell maturation antigen, expressed on multiple myeloma, licensed the binder from Jim Kochenderfer at the US National Cancer Institute and completed pre-clinical studies. We dosed our first patient with refractory myeloma with bb2121 CAR-T cells in 2016, and the initial results showed promising efficacy and a manageable safety profile (Raje, N. N. Engl. J. Med. 380, 1726-1737; 2019). Since then, we have completed treatment in this first study and a second pivotal trial, KarMMa, and are aiming toward submission to the US Food and Drug Administration for regulatory approval. Reflecting on my journey through the trials and tribulations of CAR-T cell research over the last two decades, I realize it has been quite a ride.

\section{Kristen Hege $\mathrm{e}^{1,2}$}

${ }^{1}$ Translational Medicine, Hematology/Oncology, Celgene, San Francisco, CA, USA. ${ }^{2}$ Department of Medicine, Division of Hematology/Oncology, University of California, San Francisco, CA, USA. e-mail:khege@celgene.com

Published online: 6 August 2019 https://doi.org/10.1038/s41591-019-0538-8 